\title{
Exercise Training Post Cardiac Resynchronization Therapy Improves Exercise Tolerance and Quality of Life
}

\author{
Amr Kamal*, Soha Nazmy, Mostafa Nawar, Mahmoud Hassanein \\ Cardiology Department, Faculty of Medicine, Alexandria University, Alexandria, Egypt \\ Email: *AMR.KAMAL@ALEXMED.EDU.EG
}

How to cite this paper: Kamal, A., Nazmy, S., Nawar, M. and Hassanein, M. (2021) Exercise Training Post Cardiac Resynchronization Therapy Improves Exercise Tolerance and Quality of Life. World Journal of Cardiovascular Diseases, 11, 305-318. https://doi.org/10.4236/wjcd.2021.116030

Received: May 7, 2021

Accepted: June 27, 2021

Published: June 30, 2021

Copyright $\odot 2021$ by author(s) and Scientific Research Publishing Inc. This work is licensed under the Creative Commons Attribution International License (CC BY 4.0).

http://creativecommons.org/licenses/by/4.0/

\section{(c) (i) Open Access}

\begin{abstract}
Background: Cardiac resynchronization therapy (CRT) results in improved morbidity, mortality, symptoms, quality of life (QOL) and exercise capacity, in appropriate chronic heart failure (CHF) patients. Moreover, combined exercise training (ET) and CRT maximize these improvements in these patients. The study evaluated the effect of ET on these patients in terms of QOL, functional class, exercise capacity and left ventricular ejection fraction (LVEF). Results: There were significant improvements in the QOL, functional class, exercise capacity, and LVEF compared with the Control Group. Comparison of both groups confirmed the cumulative effects of ET with CRT. The QOL improved by the end of training in the exercise group $(\mathrm{p}=0.001)$, compared to the Control Group $(\mathrm{p}=0.850)$. NYHA functional class improved significantly in the Exercise Group $(\mathrm{p}=0.013)$. Percent-predicted peak oxygen consumption $\left(\mathrm{VO}_{2}\right.$ peak) had significantly improved in the trained $(\mathrm{p}<$ $0.001)$ versus the untrained CRT Group ( $\mathrm{p}=0.596)$. There was a mean percent rise of the ejection fraction from $39.2 \pm 12.86$ to $44.40 \% \pm 14.42 \%$ in the Exercise Group compared to a non-significant change in the Control Group. Conclusion: ET in resynchronized CHF patients is feasible and further enhances QOL and exercise tolerance in addition to the improvements seen after CRT. The study therefore recommends for the prescription of ET after implantation in order to maximize the expected benefit.
\end{abstract}

\section{Keywords}

Chronic Heart Failure, Cardiac Resynchronization Therapy, Exercise

Training, Quality of Life

\section{Background}

Heart Failure (HF) presents as a disease syndrome and is caused by structural or 
functional abnormalities in the heart. It leads to compromised ventricular filling or ejection, referred to as systolic HF and diastolic HF, respectively, with each condition having distinct pathophysiology [1] [2]. Due to the ensuing inadequacy in the supply of oxygenated blood to end organs and peripheral tissues, heart failure syndrome manifests as a systemic disease affecting multiple organs. The condition is often exacerbated by neurohormonal compensatory mechanisms that try to restore cardiac function but turn out as counterproductive [3]. HF can be acute (AHF) or chronic (CHF) based on the time of onset. With over five million people in the United States suffer from CHF, and the survival rate after five years of initial diagnosis being less than 50\%, CHF is a major public health concern as per the Framingham Heart study reports [4] [5].

In addition to progressive decline in cardiac contractility, 30\% of HF patients have abnormalities in the electrical conduction system, leading to inter or intraventricular dyssynchrony. Together, they contribute to heightened morbidity, mortality and sudden death rates associated with HF. While the left bundle branch block is a known surrogate marker for LV mechanical dyssynchrony, QRS duration also influences ventricular efficiency [6]. Implantable electrical therapy, such as cardiac resynchronization therapy (CRT), is extensively used for the treatment of cardiac arrhythmias, LV dyssynchrony as well as for drug-refractory HF. Unlike the conventional pacemakers, which pace only the RV, CRT using the Biventricular pacemaker coordinates the pumping action of both the ventricles [7]. An additional built-in implantable cardioverter defibrillator, referred to as a cardiac resynchronization therapy defibrillator (CRT-D), also serves to re-establish rhythm harmony and cardiac output. However, according to the "muscle hypothesis" of CHF, disease progression is mainly due to skeletal myopathy-induced ergoreflex mechanisms that lead to neuroendocrine activation and peripheral vasoconstriction. This accounts for a probable delay in improvement even after attempted hemodynamic recovery with drugs or surgery. Non-hemodynamic modes of remedy involving Cardiac Rehabilitation with judicial exercise training can, hence, reverse muscle pathophysiology, alleviating the symptoms of cardiac failure [8] [9] [10]. CR program is scheduled into 3 phases, and it majorly involves physician advised exercise, education and counselling and behavioral interventions aimed towards risk factor modification and management associated with HF [11].

Therapeutic interventions such as cardiac rehabilitation mainly include structured exercise [12]. Structured exercise training (ET) and physical activity are recommended for cardiac health in the HF guidelines of the European Society of Cardiology. Apart from reversing peripheral abnormalities, ET can enhance mitochondrial density, oxidative function, and mediate muscle repair and LV remodeling in patients with HF. ET is recommended to all stable CHF patients irrespective of NYHA class, etiology, left ventricular ejection fraction (LVEF) or medication.

ET can be implemented taking into consideration the following aspects: 
training protocol identification, appropriate patient selection, intensity and progressive monitoring.

Hence, this study investigated the effects of ET on the capacity to exercise and quality of life (QOL) in CHF patients post CRT.

\section{Patients}

In this study, we worked with $20 \mathrm{CHF}$ patients following CRT. In our study, we had 18 men and 2 females, aged between 45 to 72 years. All the patients were stable and on full medical treatment before the study. The patients were then randomized to two equal groups:

1) Exercise Group (Group I) $(\mathrm{n}=10)$.

2) Control Group (Group II) $(\mathrm{n}=10)$.

Those patients who had no contraindications to exercise testing or training and noncardiac physical limitations were included in the study.

\section{Methods}

Patients were assessed thoroughly before their enrollment and participation in the study. Detailed pre- and post-assessments, including complete history taking, full clinical examination and resting transthoracic echocardiogram. QOL and functional class scores were recorded for all patients (Table 1), were done QOL was examined using the Minnesota Living with Heart Failure Questionnaire (MLHFQ). The responses of patients to a series of 21 questions evaluate the impact of HF and treatment on various aspects of their life and its quality, including the effectiveness of the treatment in ameliorating the symptoms, psychosocial and cognitive performance post diagnosis, etc.

Functional class was determined with the NYHA functional classification [13] [14] and the specific activity scale classification (SAS) [15] [16].

The treadmill exercise test involved the Naughton protocol [17] [18]. This test is preferred for deconditioned or older individuals and those patients suffering from chronic diseases [18]. It is symptom-limited and intended to continue until such signs and/or symptoms that necessitate its termination [19].

Peak oxygen consumption was $\left(\mathrm{VO}_{2}\right.$ predicted $)$ predicted using the Wasserman and Hansen regression equation based on physiological parameters as sex, age, height, weight, lifestyle and activity.

This $\mathrm{VO}_{2}$ peak prediction formula was used in the study along with a predicted value calculated by the treadmill during the stress test. The circulatory power, cardiac output (Q), and PRPP were also calculated in the study [20] [21] [22].

The Exercise Group underwent an exercise program consisting of three visits per week. The duration of this exercise program was four months.

By the end of the program, each session included (in order) [23] [24] [25]:

1) Warm-up period,

2) Flexibility (stretching) exercises, 
Table 1. Comparison between the studied groups according to demographic data, risk factors, and treatment.

\begin{tabular}{|c|c|c|c|c|c|c|}
\hline & \multicolumn{2}{|c|}{$\begin{array}{l}\text { Group I } \\
(n=10)\end{array}$} & \multicolumn{2}{|c|}{$\begin{array}{l}\text { Group II } \\
(\mathrm{n}=10)\end{array}$} & \multirow{2}{*}{$\begin{array}{c}\text { Test of } \\
\text { Sig. }\end{array}$} & \multirow{2}{*}{$\mathrm{p}$} \\
\hline & No. & $\%$ & No. & $\%$ & & \\
\hline \multicolumn{7}{|l|}{ Gender } \\
\hline Male & 9 & 90.0 & 9 & 90.0 & \multirow{2}{*}{$\chi^{2}=0.0$} & \multirow{2}{*}{${ }^{\mathrm{FE}} \mathrm{p}=1.000$} \\
\hline Female & 1 & 10.0 & 1 & 10.0 & & \\
\hline \multicolumn{7}{|l|}{ Age (years) } \\
\hline Min. - Max. & \multicolumn{2}{|c|}{$45.0-71.0$} & \multicolumn{2}{|c|}{$47.0-72.0$} & & \\
\hline Mean \pm SD & \multicolumn{2}{|c|}{$61.20 \pm 9.68$} & \multicolumn{2}{|c|}{$59.90 \pm 9.77$} & $\mathrm{t}=0.299$ & 0.768 \\
\hline Median & \multicolumn{2}{|c|}{64.50} & \multicolumn{2}{|c|}{61.50} & & \\
\hline \multicolumn{7}{|l|}{ Risk Factors: } \\
\hline \multicolumn{7}{|l|}{ HTN } \\
\hline No & 7 & 70.0 & 8 & 80.0 & \multirow{2}{*}{0.267} & \multirow{2}{*}{${ }^{\mathrm{FE}} \mathrm{p}=1.000$} \\
\hline Yes & 3 & 30.0 & 2 & 20.0 & & \\
\hline \multicolumn{7}{|l|}{ IHD } \\
\hline No & 3 & 30.0 & 1 & 10.0 & \multirow{2}{*}{1.250} & \multirow{2}{*}{${ }^{\mathrm{FE}} \mathrm{p}=0.582$} \\
\hline Yes & 7 & 70.0 & 9 & 90.0 & & \\
\hline \multicolumn{7}{|l|}{ DM II } \\
\hline No & 3 & 30.0 & 7 & 70.0 & \multirow{2}{*}{3.200} & \multirow{2}{*}{0.074} \\
\hline Yes & 7 & 70.0 & 3 & 30.0 & & \\
\hline OHD & 6 & 85.7 & 1 & 33.3 & \multirow{2}{*}{2.744} & \multirow{2}{*}{${ }^{\mathrm{FE}} \mathrm{p}=0.183$} \\
\hline Insulin & 1 & 14.3 & 2 & 66.7 & & \\
\hline \multicolumn{7}{|l|}{ Smoker } \\
\hline Never smoked & 2 & 20.0 & 1 & 10.0 & & \\
\hline Ex-smoker & 8 & 80.0 & 6 & 60.0 & 3.298 & ${ }^{\mathrm{MC}} \mathrm{p}=0.367$ \\
\hline Smoker & 0 & 0.0 & 3 & 30.0 & & \\
\hline Family history & & & & & & \\
\hline No & 8 & 80.0 & 9 & 90.0 & 0320 & $\mathrm{FE}_{\mathrm{n}}-1000$ \\
\hline Yes & 2 & 20.0 & 1 & 10.0 & 0.392 & $\mathrm{p}=1.000$ \\
\hline Treatment: & & & & & & \\
\hline ACEI/ARB & 10 & 100.0 & 10 & 100.0 & - & - \\
\hline BB & 10 & 100.0 & 10 & 100.0 & - & - \\
\hline Diuretic & 10 & 100.0 & 10 & 100.0 & - & - \\
\hline ARA & 10 & 100.0 & 10 & 100.0 & - & - \\
\hline Digoxin & 5 & 50.0 & 3 & 30.0 & 0.833 & 0.650 \\
\hline Amiodarone & 4 & 40.0 & 3 & 30.0 & 0.220 & 1.000 \\
\hline Nitrates & 2 & 20.0 & 3 & 30.0 & 0.267 & 1.000 \\
\hline Procolaran & 1 & 10.0 & 1 & 10.0 & 0.0 & 1.000 \\
\hline
\end{tabular}

$\chi^{2}$ : Value for Chi square FE: Fisher Exact test t: Student t-test. 
3) Exercise training session,

4) Breathing exercises [26] [27], and

5) Cool-down period.

The exercise intensity assessment depended on the heart rate reserve [13] [28], which approximately ranged from $40 \%$ to $70 \%$ [28]. This also correlated with a perceived exertion of 11 to 13 on the Borg scale (6 - 20) [28]. Intensity rose progressively from $40 \%$ to $50 \%$ for the first 4 weeks, $50 \%$ to $60 \%$ for the next 4 weeks and $60 \%$ to $70 \%$ for the rest of the program.

\section{Results}

The QOL had improved in the Exercise Group by the end of the study when compared to the Control Group (Figure 1). A basal mean score of $76.90 \pm 10.45$ points in the Exercise Group had decreased to $54.0 \pm 20.85$ points post training (about $35 \%$ decrease in the score) $(\mathrm{p}=0.001)$. This was compared to a mean score of $74.20 \pm 7.89$ points at baseline, becoming $73.40 \pm 15.90$ points at follow-up in the Control Group (about $2 \%$ increase in the score) $(\mathrm{p}=0.850)$. When compared to each other, a significant difference was seen in favor of exercise $(\mathrm{p}=0.031)$.

NYHA functional class and specific activity scale score improved significantly before and after exercise training in the Exercise Group, $(\mathrm{p}=0.013)$ and $(\mathrm{p}=$ 0.008 ) respectively (Figure 2).

$\mathrm{VO}_{2}$ peak had improved significantly in the trained $(\mathrm{p}<0.001)$ versus the untrained CRT Group ( $\mathrm{p}=0.596)$, thus, supporting the importance of exercise $(\mathrm{p}=$ 0.012 ). Also, there was an increase of $8.31 \mathrm{~mL} \mathrm{O} / \mathrm{kg} / \mathrm{min}$ and only $0.75 \mathrm{~mL}$ $\mathrm{O}_{2} / \mathrm{kg} / \mathrm{min}$ in the exercise group, and control group noticed, respectively (Figure 3).

Moreover, an increase in the EF due to ET was noted. A median increase of about $8 \%$ of the baseline value was noticed in the exercise group, compared to a median decrease of about $5 \%$ of the basal EF value in the control group (Figure 4).

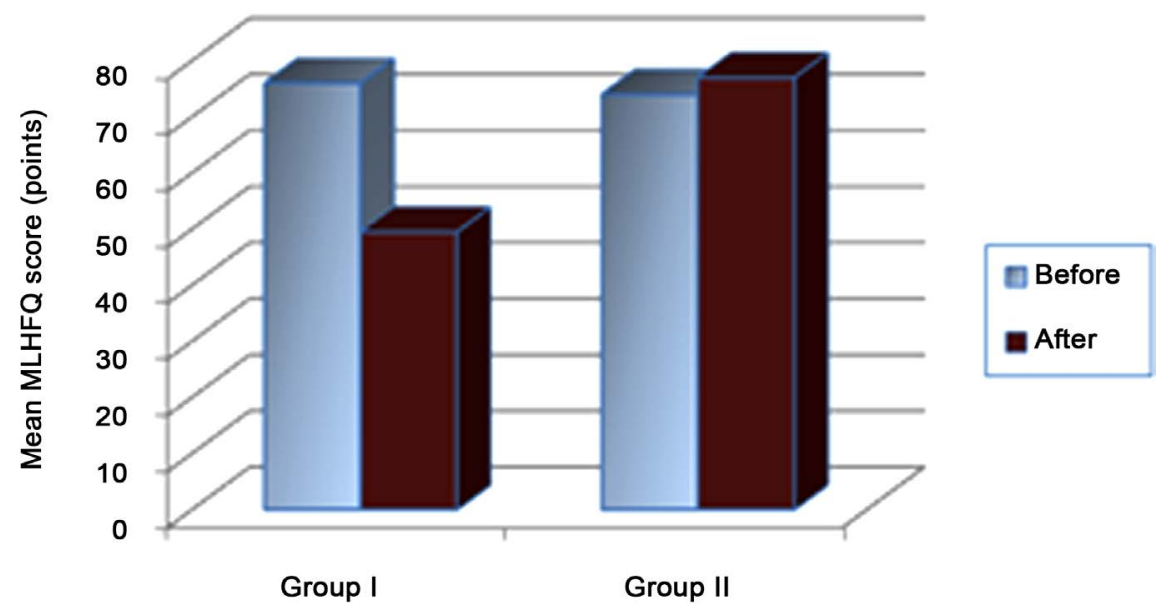

Figure 1. Comparison of MLHFQ score between Group I and Group II. 


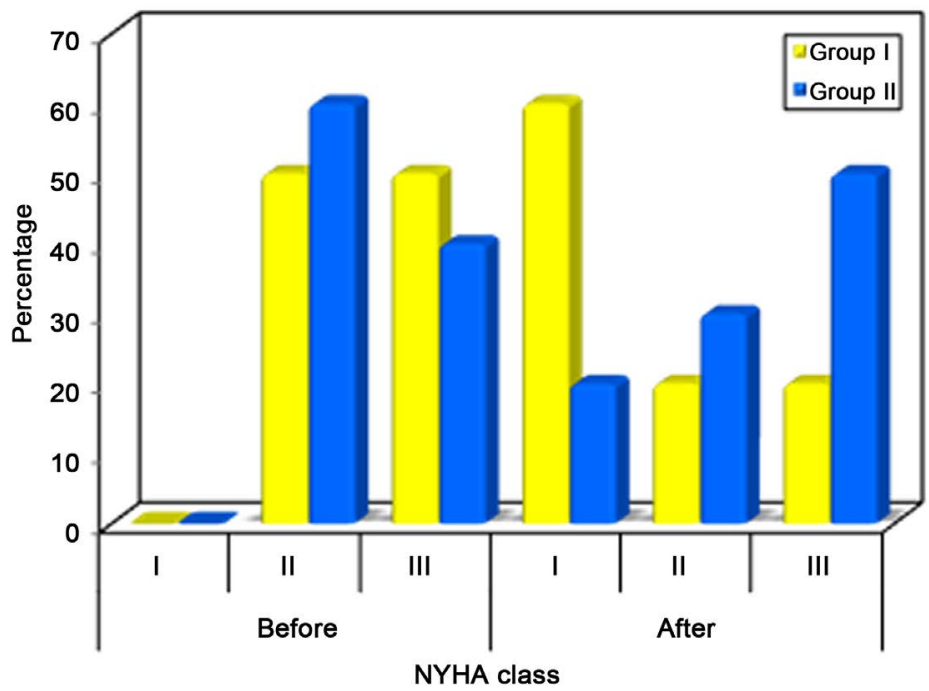

Figure 2. Comparison between Group I and Group II according to NYHA class.

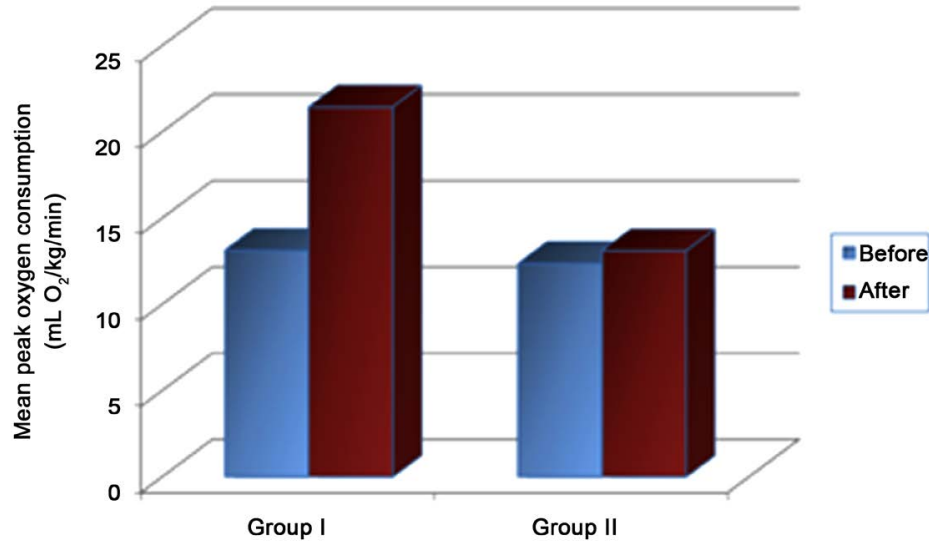

Figure 3. Mean peak oxygen consumption in Group I and Group II.

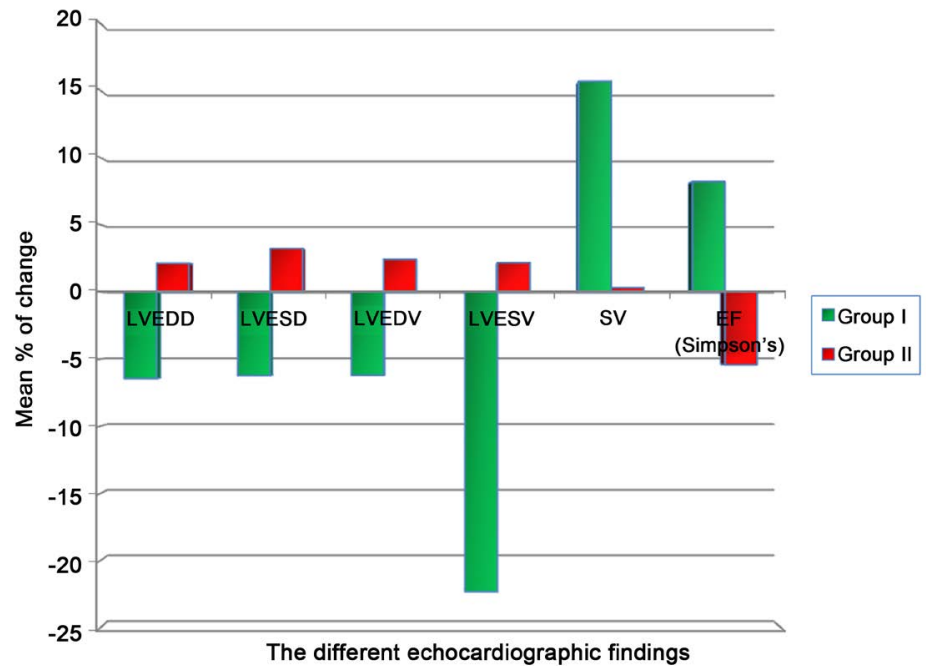

Figure 4. Comparison of echocardiographic findings relevant to exercise training in Group I and Group II. 
Table 2. Quality of life, functional class, peak oxygen consumption and exercise test duration in Group 1 and II.

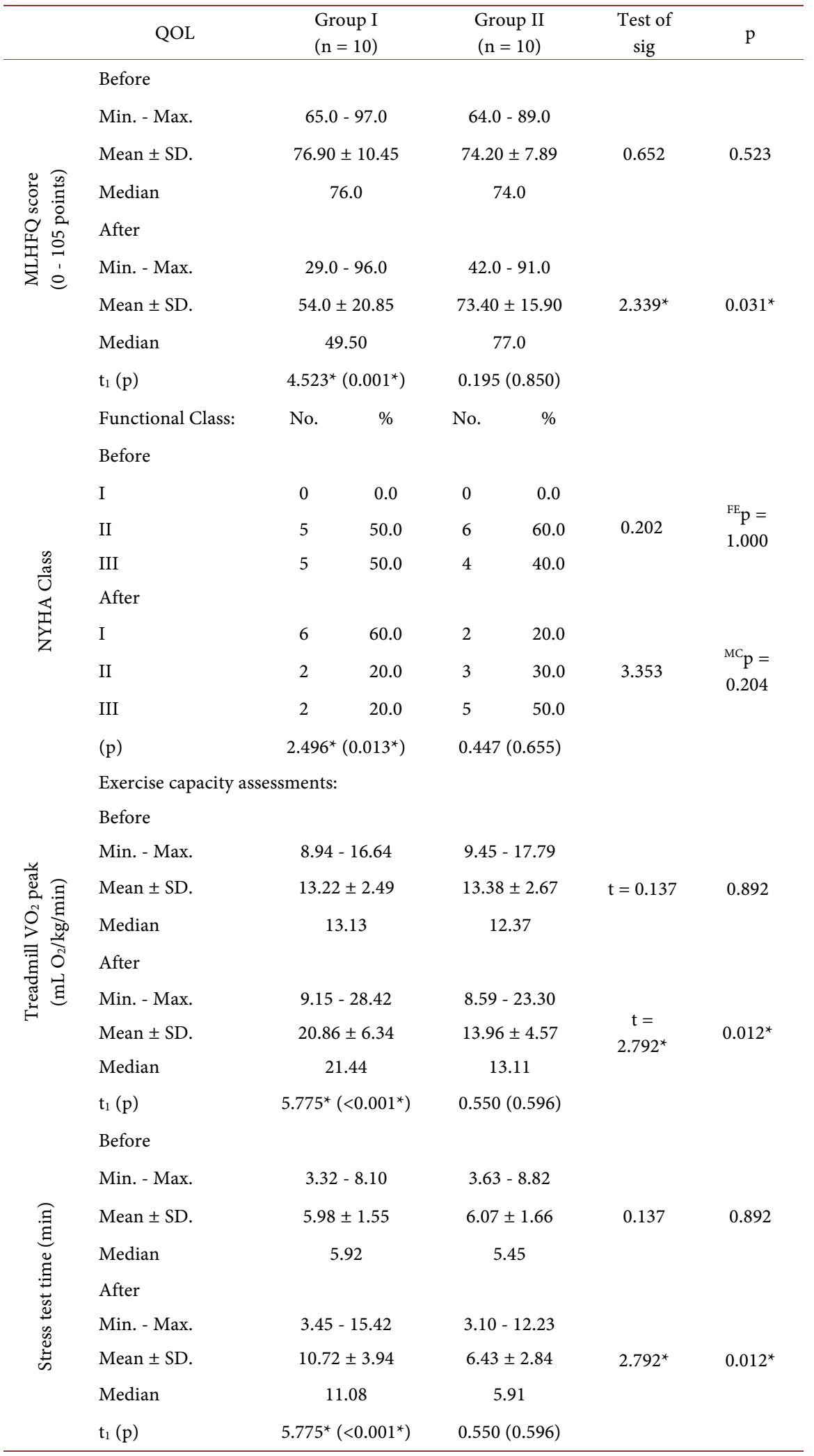

$\mathrm{t}$ : Student $\mathrm{t}$-test. $\mathrm{t}_{1}$ : Paired $\mathrm{t}$-test for comparing between before and after. ${ }^{*}$ : Statistically significant at $\mathrm{p} \leq$ 0.05 . 
Significant improvements were also observed in QOL, functional class, exercise capacity, and LVEF compared with the Control Group after four months of ET. Comparison of both groups confirmed the cumulative effects of ET with CRT (Table 2).

\section{Discussion}

In our study, a significant improvement in MLHFQ score was observed in the exercise group in comparison with the control group. The QOL for the exercise group showed a mean score of $76.90 \pm 10.45$ points before and $54.0 \pm 20.85$ points after the study. As for control group, they had a mean score of $74.20 \pm$ 7.89 points before and $73.40 \pm 15.90$ points after the study. A mean reduction of 23 points in the exercise group was seen, compared to only 1 point in the control group. Trained patients reflected an improvement in QOL after ET, compared to those in the control group. These results show and approve the importance of physical activity in these patients, and that this must be the protocol of life for them in order to have a good QOL.

Similarly, Conraads et al. reported the score reduced by 21 points in the exercise group and by 12 points in the control group, resulting in the absence of a statistical significance favoring ET between both groups at follow-up $(p=0.5)$. It must be taken into consideration that their baseline assessment was before CRT implantation (not post-CRT like our study), so the reduction of points in the control group could be linked to the positive effect of CRT. However, the further reduction of the score noticed in the exercise group supported the additional benefit of ET.

Belardinelli et al. and Patwala et al. also had a significant reduction of score in the exercise group compared to the control group. In Belardinelli's study, a mean decrease of $15 \pm 2.2$ points in the exercise group was noted, compared to a mean increase of $5 \pm 1.9$ points in the control group was noticed.

In our study, the NYHA and SAS class vastly improved in the exercise group at follow-up ( $\mathrm{p}<0.05)$, compared to the control group $(\mathrm{p}=0.655)$. Based on the NYHA classification, we had 5 patients in class II and 5 patients in class III in the exercise group at baseline. As for the control group, we had 6 patients in class II and 4 patients in class III at baseline.

At follow-up, 5 patients were in NYHA class I in the exercise group. The rest of the patients were in class II ( 3 patients) and class III ( 2 patients). On the contrary, only 1 patient was in class I in the control group, with 3 patients in class II and 6 patients in class III. However, when both groups were compared to each other, no significant difference in favor of ET was noticed ( $\mathrm{p}=0.204)$. This is most probably due to the few cases recruited in our study, since a larger number of candidates may have resulted in a significant value.

Conraads et al. had coinciding results with ours. They found the NYHA class significantly improved in the exercise group, supporting the valuable effect of ET on the functional class $(\mathrm{p}=0.04)$. When they compared both groups, they also 
noticed a non-significant difference between both groups $(\mathrm{p}=0.8)$. It should be noted that they had recruited 17 patients in their study, which is similar to the number of patients in this present study (20 patients).

Moreover, Patwala et al. showed that the NYHA class significantly improved in the exercise group $(p=0.05)$. In contrast, no change was observed in the control group $(\mathrm{p}=1)$.

Exercise intolerance being a cardinal feature of CHF and often an indication of multisystem dysfunction and disease severity, cardiopulmonary exercise testing is used for assessing the status of the condition [29].

In our study, we noticed an improvement in the mean value of the stress test duration, where it rose from $5.98 \pm 1.55$ to $10.72 \pm 3.94 \mathrm{~min}$ in the exercise group ( $\mathrm{p}<0.001)$ compared to no change in the control group $(\mathrm{p}=0.355)$. Both groups showed a statistically significant difference, supporting the value of ET was noticed $(\mathrm{p}=0.004)$.

Patwala et al. also showed similar results, where an improvement was noticed in the exercise group stress test duration rising from $9.7 \pm 3.7$ to $12.53 \pm 2.98$ min. Also, a significant difference was observed between both groups after the ET program $(\mathrm{p}<0.05)$.

In our current study, we noticed an improvement in the predicted $\mathrm{VO}_{2}$ peak between the patients before and after their ET program $(\mathrm{p}<0.001)$, and between them and the control group too $(\mathrm{p}=0.012)$. The mean $\mathrm{VO}_{2}$ peak rose from $13.22 \pm 2.49$ to $20.86 \pm 6.34 \mathrm{~mL} \mathrm{O} / \mathrm{kg} / \mathrm{min}$. As for the control group, although they had a similar baseline reading as that of the exercise group, $(13.38 \pm 2.64$ $\mathrm{mL} \mathrm{O}_{2} / \mathrm{kg} / \mathrm{min}$ ), their $\mathrm{VO}_{2}$ peak did not change much by the end of the study. Thus, an increase of $8.31 \mathrm{~mL} \mathrm{O} / \mathrm{kg} / \mathrm{min}$ in the training group compared to an increase of only $0.75 \mathrm{~mL} \mathrm{O} / \mathrm{kg} / \mathrm{min}$ in the control group was noticed in our study. This showed a significant positive difference between both groups. A relative benefit of $63 \%$ in the exercise group versus $0 \%$ in the control had been attained in this study.

Consistent with our results, Conraads et al. showed that the mean $\mathrm{VO}_{2}$ peak rose from $13.8 \pm 1.0$ to $19.3 \pm 1.2 \mathrm{~mL} \mathrm{O} / \mathrm{kg} / \mathrm{min}$, which was similar to our study. They had an increase by $5.5 \mathrm{~mL} \mathrm{O} / \mathrm{kg} / \mathrm{min}$ (i.e., $40 \%$ improvement).

Furthermore, Belardinelli et al.also showed an improvement in the $\mathrm{VO}_{2}$ peak post-exercise, where it rose by about $4.15 \mathrm{~mL} \mathrm{O} / \mathrm{kg} / \mathrm{min}$ in the exercise group (rising from $14.8 \pm 2.5$ to $18.9 \pm 2.7 \mathrm{~mL} \mathrm{O}_{2} / \mathrm{kg} / \mathrm{min}$ ), compared to a less than 1 $\mathrm{mL} \mathrm{O} / \mathrm{kg} / \mathrm{min}$ rise in the control group. Thus, improvement in the $\mathrm{VO}_{2}$ peak was more pronounced in the trained group just as with our study.

Patwala et al. also showed a significant rise of $\mathrm{VO}_{2}$ peak in their exercise group than in the control group $(\mathrm{p}<0.05)$.

Similarly, the ExTra-MATCH meta-analysis study collected data from 9 randomized ET program in which 801 patients were enrolled. Here, 2 to 12 months training improved $\mathrm{VO}_{2}$ peak from 17.2 to $20.6 \mathrm{~mL} \mathrm{O}_{2} / \mathrm{kg} / \mathrm{min}$ [30].

In agreement with our results, the Exercise in Left Ventricular Dysfunction 
and Chronic Heart Failure (ELVD-CHF) trial [31], had a baseline $\mathrm{VO}_{2}$ peak of $13.8 \pm 2.3 \mathrm{~mL} \mathrm{O} / \mathrm{kg} / \mathrm{min}$, which was similar to our study's baseline readings. Although there was an improvement by $2.4 \mathrm{~mL} \mathrm{O} / \mathrm{kg} / \mathrm{min}$ only (in the exercise group), they still supported the fact that ET improves the $\mathrm{VO}_{2}$ peak.

In the current study, at the end of the four-month follow-up period, there was a mean rise in LVEF by about $5 \%$ in the exercise group, compared to mean decrease in the value by about $2 \%$ the control group.

Similarly, Patwala et al. portrayed a mean rise in LVEF by $4.5 \%$ in the exercise group.

Additionally, the $\mathrm{EF}$ in the exercise group rose by about $5 \%$ compared to a $<1 \%$ reduction in the control group with Keyhani et al. [32].

Coinciding with our results, Conraads et al. portrayed a significant rise in EF in the exercise group after ET $(\mathrm{p}=0.02)$. It is worth mentioning that there was a smaller rise in EF of the control group (which made the comparison between both groups non-significant by the end of the study). However, as mentioned before, they had compared their results "before CRT implantation and after ET" and not "post-CRT and after ET" like our study.

\section{Conclusion}

ET significantly improves the tolerance for exercise and QOL following CRT in CHF patients. ET should be prescribed after device implantation to maximize the benefits from resynchronization therapy.

\section{Acknowledgements}

Not applicable.

\section{Conflicts of Interest}

The authors declare that they have no competing interests.

\section{Ethics Approval and Consent to Participate}

The study was conducted after the approval of the protocol by the Cardiology and Angiology Department of Alexandria Main University Hospital, and the Committee of Ethics and Research in the Faculty of Medicine, Alexandria University. The reference number is not applicable. A written consent was obtained from the participants.

\section{Consent for Publication}

Not applicable.

\section{Availability of Data and Material}

The datasets used and/or analyzed during the current study are available from the corresponding author on reasonable request. 


\section{Funding}

Not applicable.

\section{Authors' Contribution}

Dr. AK contributed as the first author of the idea and data processing and methodology as well as interpretation of the results and writing of the manuscript; Dr. SN contributed in data collection; Dr. MN contributed in data revision; Dr. $\mathrm{MH}$ contributed in data processing and revision. All authors have read and approved the manuscript.

\section{References}

[1] Inamdar, A.A. and Inamdar, A.C. (2016) Heart Failure: Diagnosis, Management and Utilization. Journal of Clinical Medicine, 5, 62. https://doi.org/10.3390/jcm5070062

[2] Fine, N.M. (2010) Heart Failure (HF) (Congestive Heart Failure). MSD Manual.

[3] Kırali, K., Özer, T. and Özgür, M.M. (2017) Pathophysiology in Heart Failure. Intech Open. https://doi.org/10.5772/66887

[4] Haennel, R.G. (2012) Exercise Rehabilitation for Chronic Heart Failure Patients with Cardiac Device Implants. Cardiopulmonary Physical Therapy Journal, 23, 23-28. https://doi.org/10.1097/01823246-201223030-00004

[5] Levy, D., Kenchaiah, S., Larson, M.G., Benjamin, E.J., Kupka, M.J., Ho, K.K.L., et al. (2002) Long-Term Trends in the Incidence of and Survival with Heart Failure. The New England Journal of Medicine, 347, 1397-402. https://doi.org/10.1056/NEJMoa020265

[6] Linde, C., Ellenbogen, K. and McAlister, F.A. (2012) Cardiac Resynchronization Therapy (CRT): Clinical Trials, Guidelines, and Target Populations. Heart Rhythm, 9, S3-S13. https://doi.org/10.1016/j.hrthm.2012.04.026

[7] Gould, P.A., Mariani, J.A. and Kaye, D.M. (2006) Biventricular Pacing in Heart Failure: A Review. Expert Review of Cardiovascular Therapy, 4, 97-109. https://doi.org/10.1586/14779072.4.1.97

[8] Coats, A.J.S., Clark, A.L., Piepoli, M., Volterrani, M. and Poole-Wilson, P.A. (1994) Symptoms and Quality of Life in Heart Failure: The Muscle Hypothesis. British Heart Journal, 72, S36-S39. https://doi.org/10.1136/hrt.72.2 Suppl.S36

[9] Piepoli, M.F. and Coats, A.J.S. (2013) The "Skeletal Muscle Hypothesis in Heart Failure” Revised. European Heart Journal, 34, 486-488. https://doi.org/10.1093/eurheartj/ehs463

[10] Coats, A.J.S. (1996) The "Muscle Hypothesis" of Chronic Heart Failure. The Journal of Molecular and Cellular Cardiology, 28, 2255-2262. https://doi.org/10.1006/jmcc.1996.0218

[11] Shah, N.P., AbuHaniyeh, A. and Ahmed, H. (2018) Cardiac Rehabilitation: Current Review of the Literature and Its Role in Patients with Heart Failure. Current Treatment Options in Cardiovascular Medicine, 20, Article No. 12. https://doi.org/10.1007/s11936-018-0611-5

[12] Delaney, J.P., Kelly, J. and Sandercock, G. (2005) The Effects of Cardiac Rehabilitation on Heart Rate Variability in Patients with Coronary Heart Disease. The British Journal of Cardiology, 12, 368-370. 
[13] Piepoli, M.F., Conraads, V.M., Corra, U., Dickstein, K., Francis, D.P., Jaarsma, T., et al. (2011) Exercise Training in Heart Failure: From Theory to Practice. A Consensus Document of the Heart Failure Association and the European Association for Cardiovascular Prevention and Rehabilitation. European Journal of Heart Failure, 13, 347-357. https://doi.org/10.1093/eurjhf/hfr017

[14] McMurray, J.J.V., Adamopoulos, S., Anker, S.D., Auricchio, A., Bohm, M., Dickstein, K., et al. (2012) ESC Guidelines for the Diagnosis and Treatment of Acute and Chronic Heart Failure 2012. The Task Force for the Diagnosis and Treatment of Acute and Chronic Heart Failure 2012 of the European Society of Cardiology. Developed in Collaboration with the Heart Failure Association (HFA) of the ESC. European Heart Journal, 33, 1787-1847. https://doi.org/10.1093/eurheartj/ehs104

[15] Conraads, V.M., Vanderheyden, M., Paelinck, B., Verstreken, S., Blankoff, I., Miljoen, H., et al. (2007) The Effect of Endurance Training on Exercise Capacity Following Cardiac Resynchronization Therapy in Chronic Heart Failure Patients: A Pilot Trial. European Journal of Cardiovascular Prevention and Rehabilitation, 14, 99-106. https://doi.org/10.1097/HJR.0b013e32801164b3

[16] Patwala, A.Y., Woods, P.R., Sharp, L., Goldspink, D.F., Tan, L.B., Wright, D.J., et al. (2009) Maximizing Patient Benefit from Cardiac Resynchronization Therapy with the Addition of Structured Exercise Training: A Randomized Controlled Study. Journal of the American College of Cardiology, 53, 2332-2339.

https://doi.org/10.1016/j.jacc.2009.02.063

[17] Williams, M.A. (2004) Special Considerations. In: Williams, M.A., Ed., Guidelines for Cardiac Rehabilitation and Secondary Prevention Programs: AACVPR (American Association of Cardiovascular and Pulmonary Rehabilitation), 4th Edition, Human Kinetics, Champaign, IL, 151-156.

[18] Belardinelli, R., Georgiou, D., Cianci, G. and Purcaro, A. (1999) Randomized, Controlled Trial of Long-Term Moderate Exercise Training in Chronic Heart Failure: Effects on Functional Capacity, Quality of Life and Clinical Outcome. Circulation, 99, 1173-1182. https://doi.org/10.1161/01.CIR.99.9.1173

[19] O’Connor, C.M., Whellan, D.J., Lee, K.L., Keteyian, S.J., Cooper, L.S., Ellis, S.J., et al. (2009) Efficacy and Safety of Exercise Training in Patients with Chronic Heart Failure. HF-ACTION Randomized Controlled Trial. JAMA, 301, 1439-1450. https://doi.org/10.1001/jama.2009.454

[20] Sullivan, M.J., Higginbotham, M.B. and Cobb, F.R. (1988) Exercise Training in Patients with Severe Left Ventricular Dysfunction. Hemodynamic and Metabolic Effects. Circulation, 78, 506-515. https://doi.org/10.1161/01.CIR.78.3.506

[21] Goldman, L., Hashimoto, B., Cook, F. and Loscalzo, A. (1981) Comparative Reproducibility and Validity of Systems for Assessing Cardiovascular Functional Class: Advantages of a New Specific Activity Scale. Circulation, 64, 1227-1234. https://doi.org/10.1161/01.CIR.64.6.1227

[22] Chung, E.K. (1983) Protocol for the Exercise ECG Test. In: Chung, E.K., Ed., Exercise Electrocardiography Practical Approach, 2nd Edition, Williams \& Wilkins, Baltimore, London, 119-128.

[23] Balady, G.J. and Morise, A.P. (2015) Exercise Testing. In: Mann, D.L., Zipes, D.P., Libbby, P., Bonow, R.O. and Braunwald, E., Eds., Braunwalds Heart Disease: $A$ Textbook of Cardiovascular Medicine, 10th Edition, Elsevier Saunders, Philadelphia, 155-171.

[24] Cohen-Solal, A., Tabet, J.Y., Logeart, D., Bourgoin, P., Tokmakova, M. and Dahan, M. (2002) A Non-Invasively Determined Surrogate of Cardiac Power ("Circulatory Power") at Peak Exercise Is a Powerful Prognostic Factor in Chronic Heart Failure. 
European Heart Journal, 23, 806-814. https://doi.org/10.1053/euhj.2001.2966

[25] Jackson, C.E. (1955) Nomogram for Simple Calculation of Cardiac Output. Circulation, 11, 635-637. https://doi.org/10.1161/01.CIR.11.4.635

[26] Papathanasiou, G., Tsamis, N., Georgiadou, P. and Adamopoulos, S. (2008) Beneficial Effects of Physical Training and Methodology of Exercise Prescription in $\mathrm{Pa}$ tients with Heart Failure. Hellenic Journal of Cardiology, 49, 267-277.

[27] Fletcher, G.F., Ades, P.A., Kligfield, P., Arena, R., Balady, G.J., Bittner, V.A., et al. (2013) Exercise Standards for Testing and Training: A Statement for Healthcare Professionals from the American Heart Association. AHA Medical/Scientific Statement. Circulation, 128, 873-934. https://doi.org/10.1161/CIR.0b013e31829b5b44

[28] Lin, S.J., McElfresh, J., Hall, B., Bloom, R. and Farrell, K. (2012) Inspiratory Muscle Training in Patients with Heart Failure: A Systemic Review. Cardiopulmonary Physical Therapy Journal, 23, 29-36. https://doi.org/10.1097/01823246-201223030-00005

[29] Kavanagh, T. (2006) Chronic Heart Failure. In: Hamm, L.F., Berra, K. and Kavanagh, T., Eds., AAVCPR (American Association of Cardiovascular and Pulmonary Rehabilitation) Cardiac Rehabilitation Resource Manual, Human Kinetics, Champaign, IL, 145-147.

[30] Auricchio, A., Stellbrink, C., Sack, S., Block, M., Vogt, J., Bakker, P., et al. (1999) The Pacing Therapies for Congestive Heart Failure (PATH-CHF) Study: Rationale, Design, and Endpoints of a Prospective Randomized Multicenter Study. The American Journal of Cardiology, 83, 130-135. https://doi.org/10.1016/S0002-9149(98)01014-5

[31] Tavazzi, L., Giannuzzi, P., Dubach, P., Opasich, C., Myers, J., Perk, J., et al. (2001) Recommendations for Exercise Testing in Chronic Heart Failure Patients. Working Group on Cardiac Rehabilitation \& Exercise Physiology and Working Group on Heart Failure of the European Society of Cardiology. European Heart Journal, 22, $37-45$.

[32] Keyhani, D., Kargarfard, M., Sarrafzadegan, N. and Sadeghi, M. (2013) Autonomic Function Change Following a Supervised Exercise Program in Patients with Congestive Heart Failure. ARYA Atherosclerosis, 9, 150-156. 


\section{List of Abbreviations}

CRT: Cardiac resynchronization therapy

QOL: quality of life

CHF: chronic heart failure

AHF: acute heart failure

ET: exercise training

LVEF: left ventricular ejection fraction

CRT-D: cardiac resynchronization therapy defibrillator

MLHFQ: Minnesota Living with Heart Failure Questionnaire

NYHA: New York Heart Association 